\title{
Richness and composition of gall-inducing arthropods at Coiba National Park, Panama
}

\author{
José Luis Nieves-Aldrey ${ }^{1}$, Alicia Ibáñez ${ }^{2} \&$ Enrique Medianero ${ }^{3}$
}

1. Museo Nacional de Ciencias Naturales (CSIC), Dpto. Biodiversidad. C/José Gutiérrez Abascal 2, 28006 Madrid (España); aldrey@mncn.csic.es. Correspondence author

2. Smithsonian Tropical Research Institute, Balboa, Republic of Panama; ibaneza@si.edu

3. Programa Centroamericano de Maestría en Entomología, Vicerrectoría de Investigación y Postgrado, Universidad de Panamá. Dirección actual: Departamento de Biodiversidad y Biología Evolutiva. Museo Nacional de Ciencias Naturales. Madrid, España; mcnem823@mncn.csic.es, medianero@yahoo.com

\section{Received 18-V-2007. Corrected 16-V-2007. Accepted 31-VII-2008.}

\begin{abstract}
Interest in studying galls and their arthropods inducers has been growing rapidly in the last two decades. However, the Neotropical region is probably the least studied region for gall-inducing arthropods. A study of the richness and composition of gall-inducing arthropods was carried out at Coiba National Park in the Republic of Panama. Field data come from samples obtained between August 1997 and September 1999, with three (two-week long) more intensive samplings. Seventeen sites, representing the main land habitats of Coiba National Park were surveyed. 4942 galls of 50 insect and 9 mite species inducing galls on 50 vascular plants from 30 botanical families were colleted. $62.7 \%$ of the galls were induced by gall midges (Diptera, Cecidomyiidae), $15.3 \%$ by mites, Eriophyidae, $8.5 \%$ by Homoptera, Psyllidae, $6.8 \%$ by Coccidae and $5.1 \%$ by Phlaeothripidae (Tysanoptera). The host plant families with the most galls were Myrtaceae with seven, Bignoniaceae with five and Euphorbiaceae, Fabaceae and Melastomataceae with four. Leaf galls accounted for about $93 \%$ of collected galls. Most leaf galls were pit/blister galls followed by covering and pouch galls. Gall richness per collecting site was between 1 and 19 species. Coiba's gall diversity is discussed in relation to data available from other tropical sites from continental Panama and the Neotropical region. Our results support the idea that it may be premature to conclude that species richness of gall inducers declines near the equator. Rev. Biol. Trop. 56 (3): 1269-1286. Epub 2008 September 30.
\end{abstract}

Key words: Coiba, gall-inducing insects, gall richness, host plant, insect/plant interaction, Panama.

Many groups of insects and mites, estimated in a range from 21000 to 211000 species, with an average of 132930 species (EspíritoSanto and Fernandes 2007), are capable of inducing galls on plants, which are structures that involve active differentiation and growth of plant tissues providing shelter, nutrition and protection for the gall-inducing organism (Meyer 1987, Nieves-Aldrey 1998, Stone and Schönrogge 2003).

Galls and gall-inducing arthropods have been the object of research for the last three centuries by dozens of naturalists and scientists worldwide (Mani 1992, see Raman et al. 2005 for a recent synthesis). Earlier information was compiled in a classic work by Houard (19081913, 1922, 1933, 1940). The most important groups of gall-inducing insects are Diptera (Cecidomyiidae), Homoptera (Cicadoidea, Psylloidea, Aphidoidea and Coccoidea), Thysanoptera and the hymenopteran families Tenthredinidae and Cynipidae. Mites inducing galls belong mostly to the family Eriophyidae. These galling-insect groups have different distribution patterns, and their distribution may be correlated with the breaking up and movement of continents (Gagné 1984). Geographic and climatic factors could also have determined the 
distribution patterns of gall-inducing arthropods (Schlinger 1974). The most important family of gall insects is Cecidomyiidae (Diptera), predominant in almost all zoogeographic regions, both tropical and temperate. Cynipidae and Tenthredinidae are mainly holarctic, gall-inducing thrips (Thysanoptera) are restricted mostly to Western Asia and gall-inducing psyllids and coccids are predominant in tropical regions (Felt 1940, Gagné 1984).

Interest in studying galls and their arthropod inducers has been growing rapidly in the last two decades (Raman et al. 2005). However, the Neotropical region continuous to be a poorly studied region for gall-inducing arthropods in general (Espírito-Santo and Fernandes 2007). The most important references are the classic work by Houard (1933), the book by Gagné (1994) on gall midges (Cecidomyiidae) and local studies in Brazil (Maia 2001, 2005, Gonçalves-Alvin and Fernandes 2001, Maia and Fernandes 2004, Costa De Oliveira and Maia 2005, Urso-Guiamares and Scareli-Santos 2006); Costa Rica (Hanson and Goméz-Laurito 2005); Mexico (Cuevas-Reyes et al. 2004a, 2004b) and Panama (Medianero et al 2001). Future research in the Neotropical region will likely change the biogeographical patterns assumed until now for gall inducing insects (Espírito-Santo and Fernandes 2007).

The aim of this study is to estimate the richness and composition of gall inducing arthropod species in an unexplored insular tropical habitat off the Pacific coast of Panama, and compare them with data available from continental Panama.

\section{MATERIALS AND METHODS}

Study area: The research was performed at Coiba National Park (World Heritage Site), as a part of the Joint Hispanic-Panamanian Program for National Parks. Coiba National Park is located in the Panamanian Pacific Ocean $\left(7^{\circ} 39^{\prime}-7^{\circ} 18^{\prime} \quad \mathrm{N} \& 81^{\circ} 53^{\prime}-81^{\circ} 35^{\prime} \mathrm{W}\right)$, $22 \mathrm{Km}$ from the mainland, and encompasses an area of 270125 ha, of which 53528 ha are insular and 216543 are marine. The insular area is composed of Coiba (50 314 ha), the largest island in tropical Pacific America, eight other minor islands and 30 islets. The maximum altitude in the Park is $420 \mathrm{~m}$ on Coiba Island. Annual rainfall is $3483 \mathrm{~mm}$. The yearly average humidity is $80 \%$ and the mean temperature is $25^{\circ} \mathrm{C}$. The area is characterized by an intense dry season, from December to April and a rainy season from May to November. The natural vegetation is well conserved, with about $80 \%$ forest coverage. An untouched tropical moist and wet forest occupies $60 \%$ of the park (Castroviejo 1997). The island of Coiba became separate from the mainland about 12000 to 18 000 years ago and houses many endemic species of animals and plants. The rough forested terrain has served to make the island inhospitable and preserved, but even more so the presence of a prison, consisting of some twenty camps scattered along the coastline, that was in operation from 1918 to 2004 (Castroviejo 1997, Fontal and Nieves-Aldrey 2004).

Field and laboratory work: Field data were obtained from several samples between August 1997 and September 1999 with three more intensive samples (two-week long) in August 1997, July 1998 and August 1999. The sampling method consisted of transects during not less than one hour (see Price et al. 1998). Along the selected collecting sites all plants were searched for galls. Seventeen sites representing the main terrestrial habitats in Coiba National Park were surveyed (Table 1, Fig. 1). Plants with galls were collected and photographed. Specimens of galled plants were dried and put into a herbarium collection, where plants were identified later. Samples of the galls were also stored in 70\% ethanol to allow further dissection and identification. Data of altitude and geographic position was taken in the field (Table 1).

The galls were dissected in the laboratory for adult and larval identification to family level. Gall-inducing species were separated by the external morphology of the galls, the larvae and the host plant. It is assumed in a study like this that gall morphotype is unique 
TABLE 1

List of sampling sites data at Coiba Nacional Park

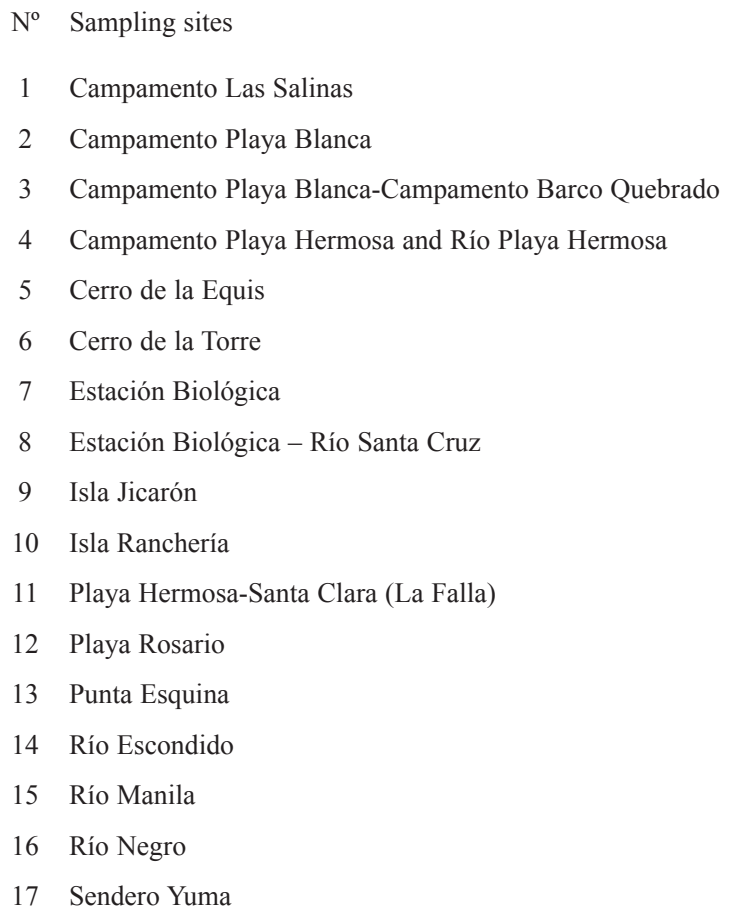

$\begin{array}{cc}\text { UTM } & \text { ALT(m) } \\ \text { 17NMU1922 } & 0-50 \\ \text { 17NMU2615 } & 0 \\ \text { 17NMU2615 } & 0 \\ \text { 17NMNO532 } & 0 \\ \text { 17NMU1841 } & 100-200 \\ \text { 17NMU0731 } & 400 \\ \text { 17NMU196429 } & 40 \\ \text { 17NMU1942-1642 } & \\ \text { 17NMJ1205 } & 0 \\ \text { 17NMU2244 } & 0-100 \\ \text { 17NMU0328 } & 100 \\ \text { 17NMU1644 } & 0 \\ \text { 17NMU2533 } & 0 \\ \text { 17NMU1341 } & 0-10 \\ \text { 17NMU1413 } & 0 \\ \text { 17NMU1720 } & 100 \\ \text { 17NMU2039 } & 0\end{array}$

Numbers represented on map of Figure 1.

for each gall-inducing species (Mani 1964, Ananthakrishnan 1984). Yet a very conservative methodology was employed (see Hanson and Goméz-Laurito 2005); galls found in petioles, in leaf blades, as well as in the nerve of a given leaf, and those without differentiation in plant species of the same genus were considered as of the same gall-inducing species.

Data analysis: Gall-inducing arthropod species richness (S) was calculated based on the number of different galls found and the alpha diversity index $(\alpha)$ was estimated (Fisher 1943):

$$
\mathrm{S}=\alpha \log \mathrm{e}(\mathrm{I}+\mathrm{N} / \alpha) .
$$

$S$ is the number of species in a sample, $N$ is the number of individuals in a sample and $\alpha$ is the diversity index. This index is not influenced by the two limitations of other known indeces because it is independent of sample size and it does not give more weight to species abundance (Wolda 1983).

To verify sampling efficiency we constructed species accumulation curves with the estimator Chao 1, Bootstrap and Jacknife 1 (100 random). The species accumulation curve is based on individual-based assessment protocol (see Gotelli and Colwell 2001). We used the software EstimatesS ${ }^{\circledR} 8$ (Colwell 2006).

\section{RESULTS}

Taxonomic and faunistic considerations: A total 4942 galls of 59 species, 50 insects and 9 mites, associated with 50 species of plants from 37 genera and 30 families were collected at Coiba National Park. It was not possible to identify the genus of nine host plant species 


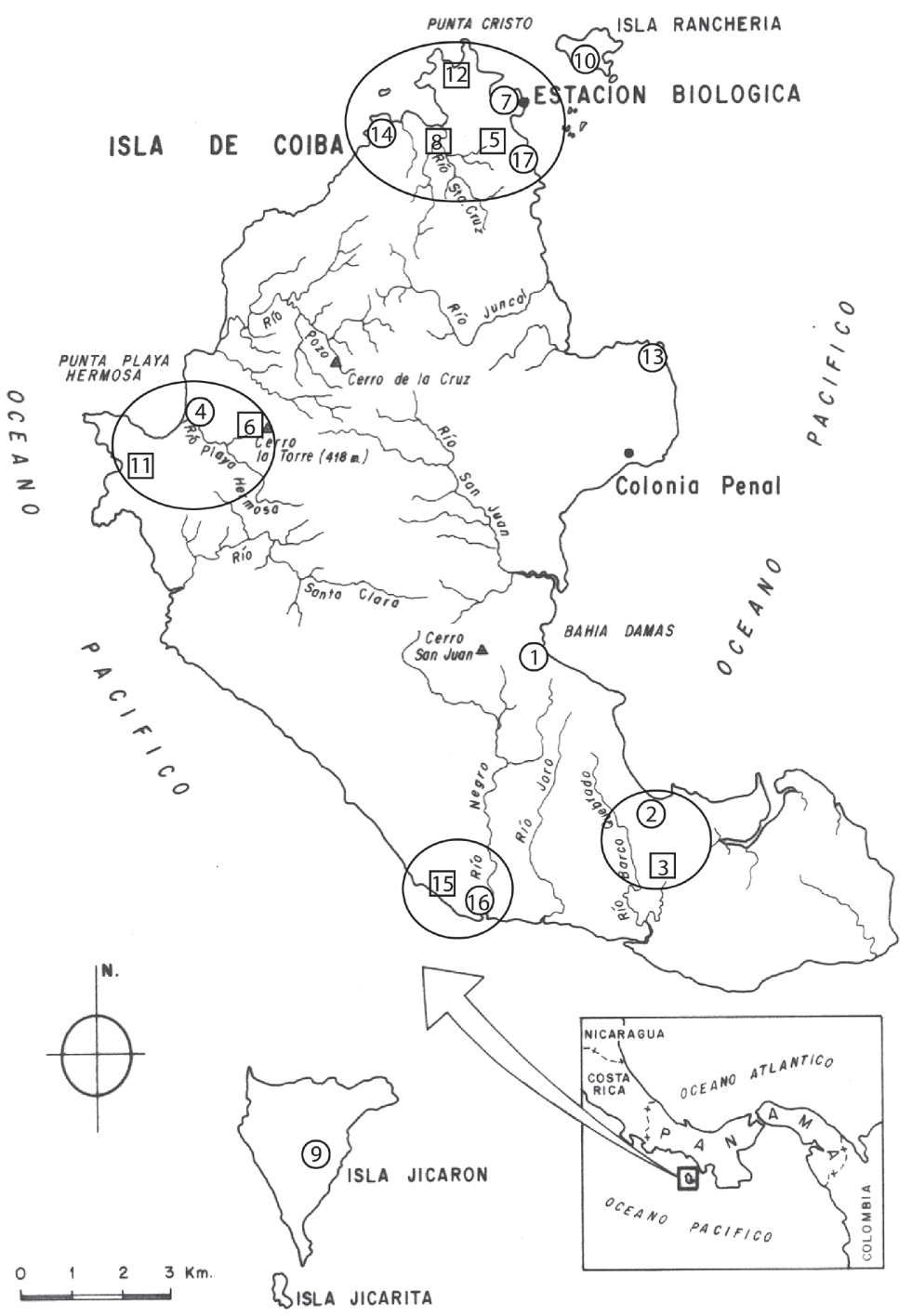

Fig. 1. Map of Coiba National Park, showing sampling sites. The four groupings correspond to the four different plant communities at Coiba Island.

mostly Myrtaceae. $62.7 \%$ of the galls were induced by species of the family Cecidomyiidae, $15.3 \%$ by Eriophyidae, $8.5 \%$ by Psyllidae, $6.8 \%$ by Coccidae, $5.1 \%$ by Phlaeothripidae; one species corresponding to $1.7 \%$ could not be determined. 93. $1 \%$ of the galls were found on leaves, $3.4 \%$ on stems and $5.2 \%$ on buds (Table 2 ). The plant families hosting the most gallinducing species were Myrtaceae with seven,
Bignoniaceae with five and Euphorbiaceae, Fabaceae and Melastomataceae with four (Table $3)$. The plant species with the greatest diversity of galls was Calophyllum longifolium (Clusiaceae) with three, seven species were found with two galls (Amphitecna latifolia, Mansoa sp., Acalypha diversifolia, Casearia commersoniana, Lacistema aggregatum, Cassipourea elliptica and Pouteria foveolata) whilst only one 
TABLE 2

Number of gall-inducing species per insect family and their affected host plant organ

Gall inducing family

Cecidomyiidae

Eriophyidae

Psyllidae

Coccidae

Phlaeothripidae

Indeterminate

$$
\text { Leaf gall }
$$

36

7

5

4

2

1
Bud gall

Stem gall

1

1

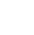

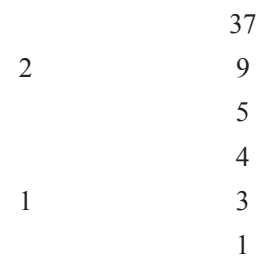

TABLE 3

Plant family and gall inducing arthropods numbers

Plant family No. of species with galls Total No. of galls Mites Midges

Psyllids Coccids Thrips

Annonaceae
Asteraceae
Bignoniaceae
Bombacaceae
Burseraceae
Celastraceae
Chrysobalanaceae
Clusiaceae
Combretaceae
Euphorbiaceae
Flacourtiaceae
Lacistemataceae
Lauraceae
Leguminosae
Melastomataceae
Moraceae
Myristicaceae
Myrsinaceae
Myrtaceae
Nyctaginaceae
Ochnaceae
Pellicieraceae
Rhamnaceae
Rhizophoraceae
Rubiaceae
Sapindaceae
Sapotaceae
Vterculiaceae
Vitaceae

Annonaceae

1

1

1

\section{1}

1
Total 
gall-inducing species was found infesting two species of Ficus. Alpha diversity was 9.4 but, species accumulation curves did not become asymptotic suggesting than there are more gallinducing species at Coiba National Park than those collected in this study. Gall-inducing species number increases with individual sample
(Fig. 2). Gall richness per collecting site was between 1 and 19 species. $71 \%$ of all species were found at only one site, $22 \%$ in two, $5 \%$ in three, $2 \%$ in four, while no species was present in more than four sites. The gall inducing arthropod community studied shows a high complementarity among collecting sites.

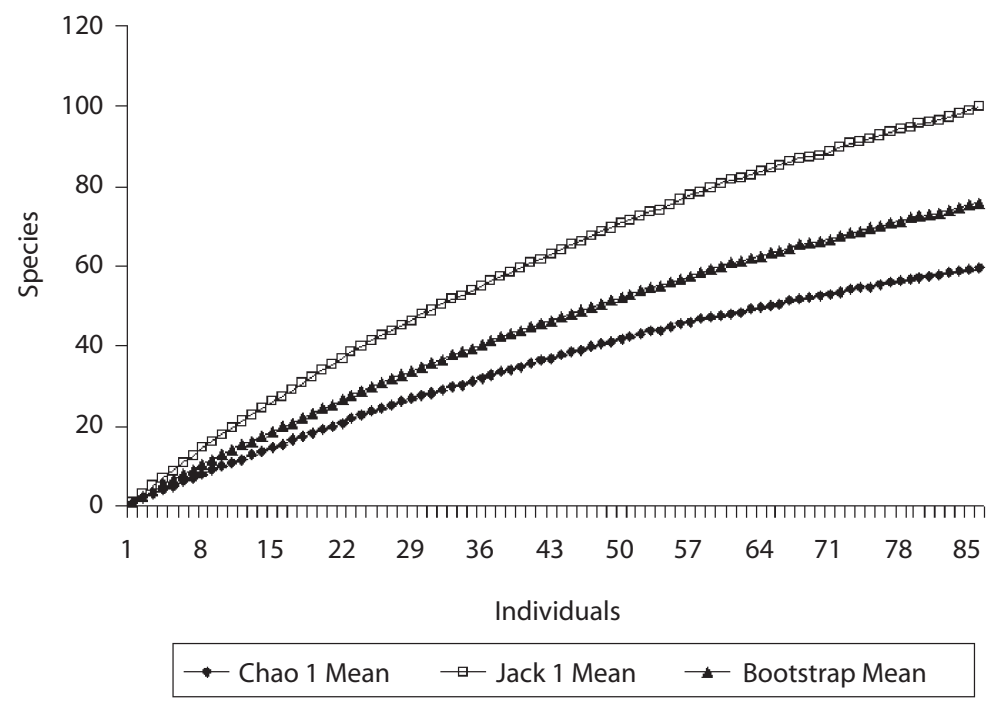

Fig. 2. Species accumulation curves with estimator Chao 1, Bootstrap and Jacknife 1 (100 random).

List of gall-inducing taxa and host plant species at Coiba National Park ( $G=$ Gall).

\section{ANNONACEAE}

Gen. Ind.

G59. (Fig. 4H). Family gall-inducer: Cecidomyiidae.

Leaf gall, ovoid with hairs on upper side.

\section{BIGNONIACEAE}

Amphitecna latifolia (Mill.) A.H. Gentry

G1 (Fig. 3A). Family gall-inducer: Cecidomyiidae

Leaf gall, spindle shaped, on mid or secondary veins, distinct on both sides of leaf.

Manglar De Sta. Cruz. 31-VIII-97. J. L. Nieves leg.

G2. Family gall-inducer: Coccidae

Leaf gall; blister more distinct on low surface.

Manglar de Sta. Cruz. 31-VIII-97. J. L. Nieves leg.

Cydista aequinoctialis (L) Miers.

G3 (Fig. 3B). Family gall-inducer: Cecidomyiidae 
Leaf gall; spindle shaped, on petiole, mid and secondary veins.

I. Jicarón. 3-X-97. A. Ibáñez leg

I. Ranchería. 7-IX-97. A. Ibáñez leg

Beach estación Biológica. 30-VIII-97. J. L. Nieves leg

Cerro Equis. 26-VIII-97. J. L. Nieves leg.

Mansoa sp.

G4. Family gall-inducer: Cecidomyiidae

Leaf gall, a blister distinct on both sides of leaf.

Sendero Yuma. IX-97. A. Ibáñez leg.

G4a. Family Gall Inducer: Cecidomyiidae

Leaf gall, a spindle shaped swelling on mid vein.

Sendero Yuma. IX-97. A. Ibáñez leg.

\section{BOMBACACEAE}

Quararibea asterolepis Pittier

G5. Family gall-inducer: Cecidomyiidae

Leaf gall, a small blister distinct on both sides of leaf.

I. Jicarón. 3-X-97. A. Ibáñez leg.

\section{BURSERACEAE}

Tetragastris panamensis (Engl.) Kuntze

G6. Family gall-inducer: Psyllidae

Leaf gall, small circular blister more distinct on upper side; on lower side appear as a pit or depression.

Cerro Equis XI-97. A. Ibáñez leg.

Protium confusum (Rose) Pittier

G7. (Fig. 3C). Family gall-inducer: Psyllidae

Leaf gall, circular, small, distinct on upper side; a pit on low part.

I. Ranchería. 7-IX-97. A. Ibáñez leg

Cerro de la Torre. 12-VI-98. A. Ibáñez leg.

\section{CELASTRACEAE}

Maytenus schippii Lundell

G9. Family gall-inducer: Cecidomyiidae

Leaf gall, a median size blister of leaf blade distinct on both sides of leaf.

Río Santa Clara. 29-XI-97. A. Ibáñez leg.

\section{CHRYSOBALANACEAE}

Licania hypoleuca Benth.

G10. Family gall-inducer: Cecidomyiidae

Leaf gall, subspherical, small.

Playa Rosario. 22-VII-98. J. L. Nieves leg.

Licania platypus (Hemsl.) Fritsch

G11. Family gall-inducer: Cecidomyiidae

Leaf gall, on blades subspherical, small and abundant.

I. Canal De Afuera. 6-XI-97. A. Ibáñez leg. 

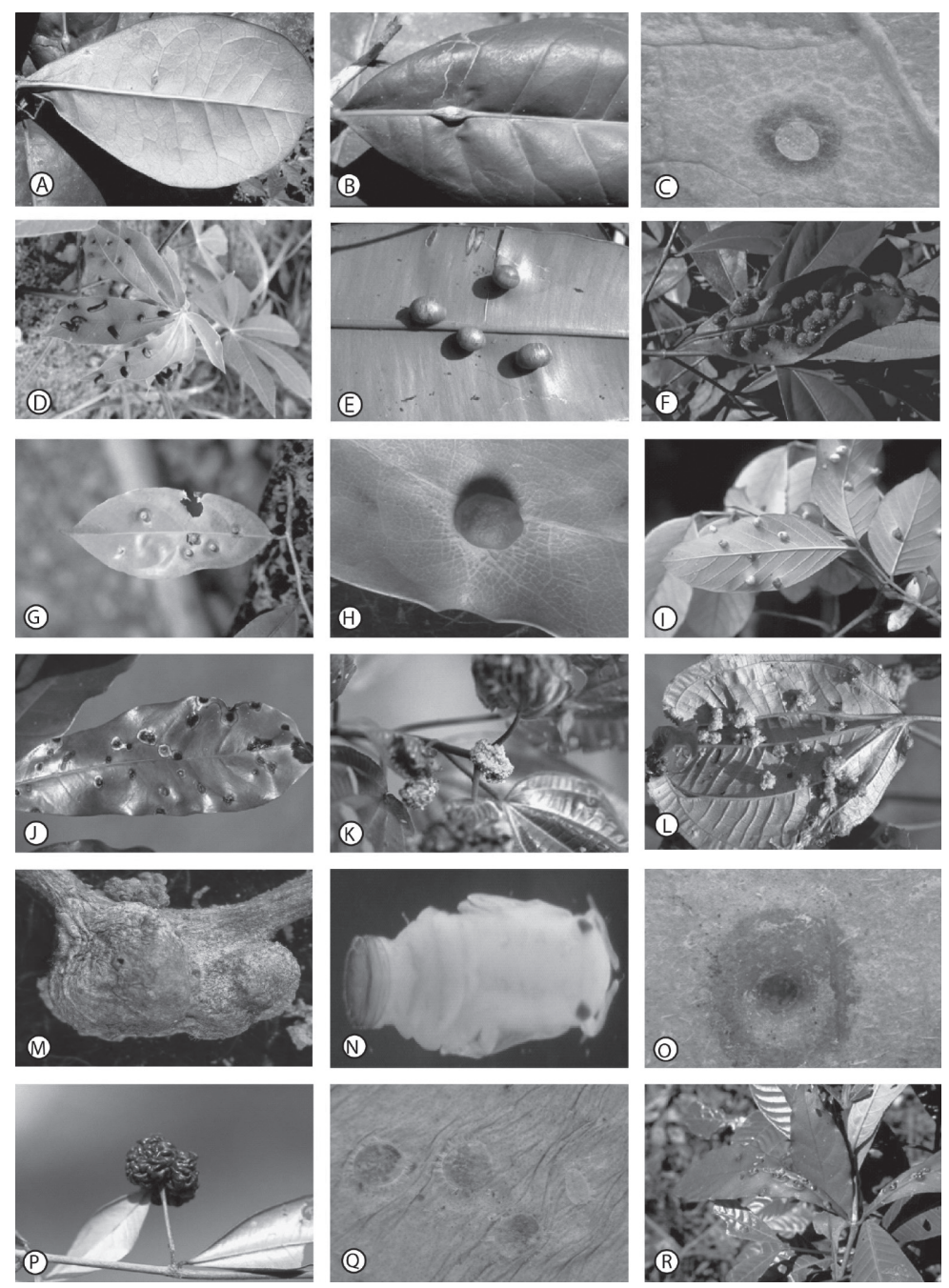

Fig. 3. Pictures of galls from Coiba: (A) gall midge on Amphitecna latifolia, (B) gall midge on Cydista aequinoctialis, (C) psyllid on Protium confusum, (D) gall midge on Manihot esculenta, (E) gall midge on Calophyllum longifolium, (F - G ) gall midge on Lacistema aggregatum, (H) psyllid on Cinnamomum triplinerve, (I) gall midge on Lonchocarpus sp., (J) gall midge on Pouteria foveolata, (K) mite gall on Miconia lacera, (L), mite on Miconia nervosa, (M) gall midge on Casearia commersoniana, (N) psyllid on Sorocea sp., (O) coccoid gall on Myrtaceae, (P) thrip gall on Myrtaceae, (Q) coccoid gall on Pelliciera rhizophorae, (R) gall midge on Psychotria horizontalis. 


\section{CLUSIACEAE}

Calophyllum longifolium Willd.

G20 (Fig. 3E). Family gall-inducer: Cecidomyiidae

Leaf gall, on blades, bean shaped, big more obvious on underside.

Playa Blanca-Cativales. 26-VIII-97. J .L. Nieves leg

Cerro De La Equis. 21-III-98. A. Ibáñez leg.

G21. Family gall-inducer: Eriophyidae

Gall leaf, on blades, tiny pocks in eyelet shape, on both sides of midrib.

Cerro De La Torre. 18-VII-98. J. L. Nieves leg.

G22. Family gall-inducer: Cecidomyiidae

Gall leaf, small pustule on blades.

Cerro De La Equis. 26-VIII-97. J. L. Nieves leg.

\section{COMBRETACEAE}

Terminalia amazonia (J.F. Gmel.) Exell

G12. Family gall-inducer: Cecidomyiidae

Leaf gall, ovoid irregular distinct on both sides of leaf.

Cerro de la Torre. 22-VII-98. J. L. Nieves leg.

\section{COMPOSITAE (ASTERACEAE)}

Gen. sp. Indet.

G13. Family gall-inducer: Phlaeotripidae

Gall a folded at tip of the leaf blades.

Sendero de Santa Cruz. 26-VIII-99. A. Ibáñez leg.

\section{EUPHORBIACEAE}

Acalypha diversifolia Jacq.

G14. Family gall-inducer: Eriophyidae

Bud gall, large, subspherical.

Rio Negro. 9-XII-97. A. Ibáñez leg

I. Jicarón. 9-XII-97. A. Ibáñez leg.

G15. Family gall-inducer: Eriophyidae

Leaf gall, a small pouch more obvious on upper side.

Rio Negro. XII-97. A. Ibáñez leg

Cerca De Sta. Cruz. XII-97. A. Ibáñez leg.

Croton schiedeanus Schltdl.

G16. (Fig. 4A).Family gall-inducer: Cecidomyiidae

Leaf gall, small blister on blades, more obvious in underside.

Manila, cerca de la parcela. 19-VI-98. A. Ibáñez leg.

Manihot esculenta Crantz

G17 (Fig. 3D). Family gall-inducer: Cecidomyiidae

Leaf gall, spindle shaped on blades, colour red, more obvious on upper side.

Playa Blanca. VIII-97. J. L. Nieves leg.

\section{FABACEAE}

Inga multijuga Benth.

G25. Family gall-inducer: Cecidomyiidae

Leaf gall, tiny pustule, more obvious on upper side.

Cerro De La Equis. 12-VI-98. A. Ibáñez leg. 
Inga sp.

G26. Family gall-inducer: Cecidomyiidae

Leaf gall, small blister.

Camino de las Salinas. 27-VIII-99. A. Ibáñez leg.

Lonchocarpus sp.

G27 (Fig. 3I). Family gall-inducer: Psyllidae

Leaf gall, ovoid, on both sides of blades, medial size.

Punta Esquina. 25-VIII-97. J. L. Nieves leg.

Prioria copaifera Griseb.

G28. (Fig. 4G). Family gall-inducer: Cecidomyiidae

Leaf gall, spherical small, abundant, scar on underside.

Playa Blanca-Cativales. 26-VIII-97. J.L.Nieves leg.

\section{FLACOURTIACEAE}

Casearia commersoniana Cambess.

G18. (Fig. 3M). Family gall-inducer: Cecidomyiidae

Gall on stems, swelling irregular, mainly on one side.

Río Negro. 9-XII-97. A. Ibáñez leg.

G19. Family gall-inducer: Cecidomyiidae

Gall on leaf, small pouch on mid vein, more obvious on upper side.

Río Negro. 9-XII-97. A. Ibáñez leg.

\section{LACISTEMATACEAE}

Lacistema aggregatum (Bergius) Rusby

G23 (Fig. 3F). Family gall-inducer: Cecidomyiidae

Leaf gall, spherical in groups, hairy, medial size.

Cerro De La Equis. 25-VIII-97. J. L. Nieves leg

G23a (Fig. 3G)

Family gall-inducer: Cecidomyiidae

Leaf gall, pit gall, cilíndrical distint on low blade of leaf.

Playa Hermosa (Falla). 20-VII-98. J. L. Nieves leg.

\section{LAURACEAE}

Cinnamomum triplinerve (Ruiz \& Pav.) Kosterm

G24 (Fig. 3H). Family gall-inducer: Psyllidae

Leaf gall, globular on secondary veins, more obvious in upper side.

Cerro De La Torre. 18-VIII-98. J. L. Nieves leg

I. Ranchería. 19-VII-98. J. L. Nieves leg

Cima del Cerro de la Torre. 7-III-98. J. L Nieves leg.

\section{MELASTOMATACEAE}

Clidemia discolor (Triana) Cogn.

G29. Family gall-inducer: Eriophyidae

Bud gall, flowers.

Manglar De Sta. Cruz. 24-VII-98. J. L. Nieves leg.

Miconia lacera (Bonpl.) Naudin

G31. (Figs. 4K, 5E) Family gall-inducer: Eriophyidae

Gall on buds and stems, subspherical.

Cerro De La Torre. 7-III-98. A. Ibáñez leg. 

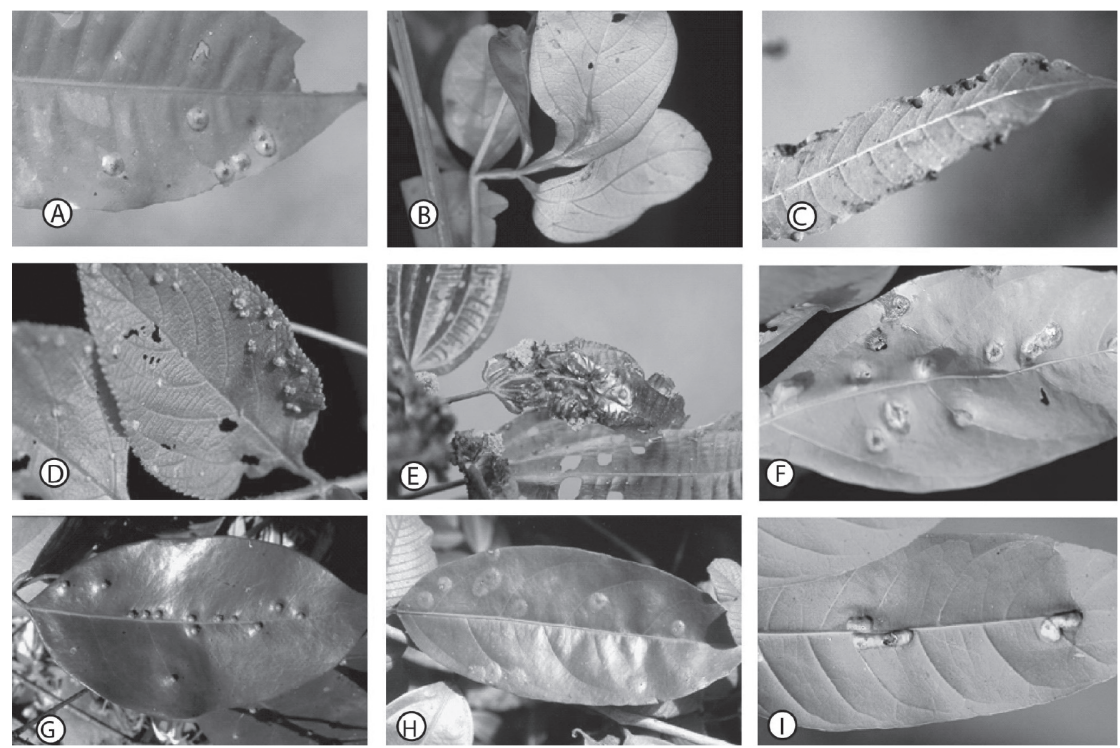

Fig. 4. Pictures of galls from Coiba: (A) gall midge on Croton schiedeanus, (B, F) gall midge on Serjania mexicana, (C) gall midge on Pouteria foveolata, (D) mite gall on Lantana camara, (E) mite gall on Miconia lacera, (G) gall midge on Prioria copaifera, (H) gall midge on Annonaceae, (I) gall midge on Faramea occidentalis.

Miconia minutiflora (Bonpl.) DC.

G32. Family gall-inducer: Eriophyidae

Dense erineum on underside of leaf, hairs red.

Cerro De La Equis. 26-VIII-97. J. L. Nieves leg

Estación Biológica. Otoño 98. A. Ibáñez leg

Sendero de Santa Cruz. 26-VIII-99. A. Ibáñez leg.

Miconia nervosa (Sm.) Triana

G30. (Fig. 3L) Family gall-inducer: Eriophyidae

Leaf gall, more obvious on underside, blades hair.

Rio Negro. 9-XII-97. A. Ibáñez leg.

Cerro De La Equis. 18-VII-98. J. L. Nieves leg.

MORACEAE

Ficus popenoei Standl.

G33. Family gall-inducer: Cecidomyiidae

Leaf gall, pouch on blades, small.

Cerro De La Equis. 21-III-98. A. Ibáñez leg.

Sorocea sp.

G35. Family gall-inducer: Psyllidae (Fig. 3N)

Leaf gall, spindle shaped, on blades abundant more obvious on upper side.

I. Ranchería. 7-IX-97. A. Ibáñez leg.

\section{MYRISTICACEAE}

Virola $\mathrm{sp}$.

G36. Family gall-inducer: Cecidomyiidae 
Leaf gall, spindle shaped, in blades, tiny.

Cerro De La Equis. 12VI-98. A. Ibáñez leg.

\section{MYRSINACEAE}

Ardisia sp.

G37. Family gall-inducer: Cecidomyiidae

Leaf gall, ovoid in blades medial size.

Manglar De Sta. Cruz. 24-VII-98. J. L. Nieves leg.

\section{MYRTACEAE}

Gen. Ind.

G38. Family gall-inducer: Cecidomyiidae

Leaf gall, ovoid, small.

Cerro De La Equis. 26-VIII-97. J. L. Nieves leg.

Gen. Ind.

G39 (Fig. 3P). Family gall-inducer: Phlaeotripidae

Bud gall on flower irregular spherical.

Manila. 23-VII-98. J .L. Nieves leg

I. Jicarón. 3-X-97. A. Ibáñez leg.

Gen. Ind. 1, Sp.3

G40 (Fig. 3O). Family gall-inducer: Coccidae

Leaf gall, blister shaped, with opening below.

Río Escondido. Otoño 97. A. Ibáñez leg.

Gen. Ind. 2, Sp. 1

G41. Family gall-inducer: Cecidomyiidae

Leaf gall subspherical irregular on blades.

La Falla. Otoño 97. A. Ibáñez leg.

Gen. Ind. 3, Sp. 4

G42. Family gall-inducer: Eriophyidae

Gall leaf, blister shaped, small more obvious on upper side.

Playa Cerro De La Equis. Otoño 97. A. Ibáñez leg. Sp. 5

G43. Family gall-inducer: Cecidomyiidae

Leaf gall, on blades, ovoid small.

Cima del Cerro De La Torre. 7-III-98. A. Ibáñez leg.

Gen. Ind. Sp. 6

G44. Family gall-inducer: Phlaeotripidae

Leaf gall, blades margin folded thick.

\section{NYCTAGINACEAE}

Neea $\mathrm{sp}$.

G45. Family gall-inducer: Cecidomyiidae

Leaf gall, blister in blades, distinct in both sides of leaf.

I. Ranchería. 19-VII-98. J. L. Nieves leg

Rio Negro. 9-XII-97. A.Ibañez leg.

OCHNACEAE

Ouratea lucens (Kunth) Engl.

G46. Family gall-inducer: Indeterminate 
Leaf gall, blister in blades.

Cerro De La Equis. VIII-97. J. L. Nieves leg

Subida a la Falla. 21-IX-99. A. Ibáñez leg.

PELLICIERACEAE

Pelliciera rhizophorae Triana \& Planch.

G47 (Fig. 3Q). Family gall-inducer: Coccidae

Leaf gall, blister small, abundant.

Manglar De Sta. Cruz. IX- 98/ 26-VIII-99. A. Ibáñez leg.

RHAMNACEAE

Gouania lupuloides (L.) Urb.

G48. Family gall-inducer: Cecidomyiidae

Leaf gall, ovoid on blades with hairs.

I. Jicarón. 30-VIII-97. J. L. Nieves leg.

\section{RHIZOPHORACEAE}

Cassipourea elliptica (Sw.) Poir.

G49. Family gall-inducer: Cecidomyiidae

Leaf gall, spindle shaped, on mid vein, distinct on both sides of blade.

Sendero Yuma. 25-VIII-97. J. L. Nieves leg

Cerro De La Equis, 21-III-98. J. L. Nieves leg.

G50. Family Gall Inducer: Coccidae

Leaf gall, ovoid small on blade more obvious on upper side.

Cerro De La Equis, 21-III-98. J. L. Nieves leg.

\section{RUBIACEAE}

Faramea occidentalis (L.) A. Rich.

G51 (Fig. 4I). Family gall-inducer: Cecidomyiidae

Leaf gall, blister shaped on mid vein more obvious on underside.

Manila. 23-VII-98. J. L. Nieves leg

I. Ranchería. 7-IX-97. A. Ibáñez leg.

Psychotria horizontalis Sw.

G52 (Fig. 3R). Family gall-inducer: Cecidomyiidae

Leaf gall, blister irregular on mid vein and petioles.

Cerro De La Equis. VIII-97. J. L. Nieves leg

Catival. 18-X-97. A. Ibáñez leg.

Cerro De La Torre. 7-III-98. A. Ibáñez leg.

\section{SAPINDACEAE}

Serjania mexicana (L.) Willd.

G53 (Fig. 4 B, F). Family gall-inducer: Cecidomyiidae

Leaf gall on blades, petioles but, mainly on mid and secondary veins.

Camino de las Salinas. 27-VIII-99. A. Ibáñez leg.

\section{SAPOTACEAE}

Pouteria cf. foveolata T.D. Penn.

G54 (Fig. 4C). Family gall-inducer: Cecidomyiidae 
Leaf gall, ovoid small, aligned on blades margin.

Manglar De Sta. Cruz. XI-97. A. Ibáñez leg

Cerro De La Equis. XI-97. A. Ibáñez leg

Cerro De La Torre. 22-VII-98. J. L. Nieves leg

Manglar De Sta. Cruz. 24-VII-98. J L. Nieves leg.

G55 (Fig. 3J). Family gall-inducer: Cecidomyiidae

Leaf gall, ovoid small on leaf blade.

Cerro De La Torre. 22-VII-98. J. L. Nieves leg

Manglar De Sta. Cruz. 24-VII-98. J L. Nieves leg.

\section{STERCULIACEAE}

Theobroma angustifolium Moç. \& Sessé ex DC.

G56. Family gall-inducer: Cecidomyiidae

Leaf gall, small spindle in leaf blade.

I. Jicarón. 3-X-97. A. Ibáñez leg.

\section{VERBENACEAE}

\section{Lantana camara L.}

G57 (Fig.5D). Family gall-inducer: Eriophyidae

Leaf gall; a small pouch on leaf blades.

Camino de las Salinas. 27-VIII-99. A. Ibáñez leg.

\section{VITACEAE}

Cissus microcarpa Vahl

G58. Family gall-inducer: Cecidomyiidae

Leaf gall, ovoid abundant, distinct in both sides of blades, medial size.

Sendero de Santa Cruz. 26-VIII-99. J. L. Nieves leg.

\section{DISCUSSION}

Gall-inducing arthropods have been more thoroughly sampled in temperate regions than in tropical regions. Indeed, from the perspective of biodiversity inventories, we have barely scratched the surface of the tropical faunas (Hanson and Goméz-Laurito 2005). A global pattern in local number of gall-inducing insects was described recently (see Price et al. 1998). The study pointed to a greater richness of gallinducing insects in warm dry regions, while temperate and tropical areas with humid, mesic vegetation showed lower gall richness indices. However, as recently acknowledged by EspíritoSanto and Fernandes (2007), these results could be affected by an under-sampling of tropical rain forests, where gall richness could be higher than predicted by Price et al. (1998).

Our results show than the moist and wet tropical forests of Coiba National Park revealed a rich diversity of gall-inducing arthropods, with species richness numbers higher than those registered in continental Panama (see Price et al. 1998) and other parts of the Neotropical region (see Cuevas et al. 2004a, Costa De Oliveira and Maia 2005) (Table 4). Although we did not collect all species in Coiba Island, the total of fifty nine species, with a range of one to nineteen gall inducer species per site, in Coiba National Park is more that the twelve proposed for mesic vegetation in the literature (see Price et al. 1998). Coiba National Park diversity is similar 


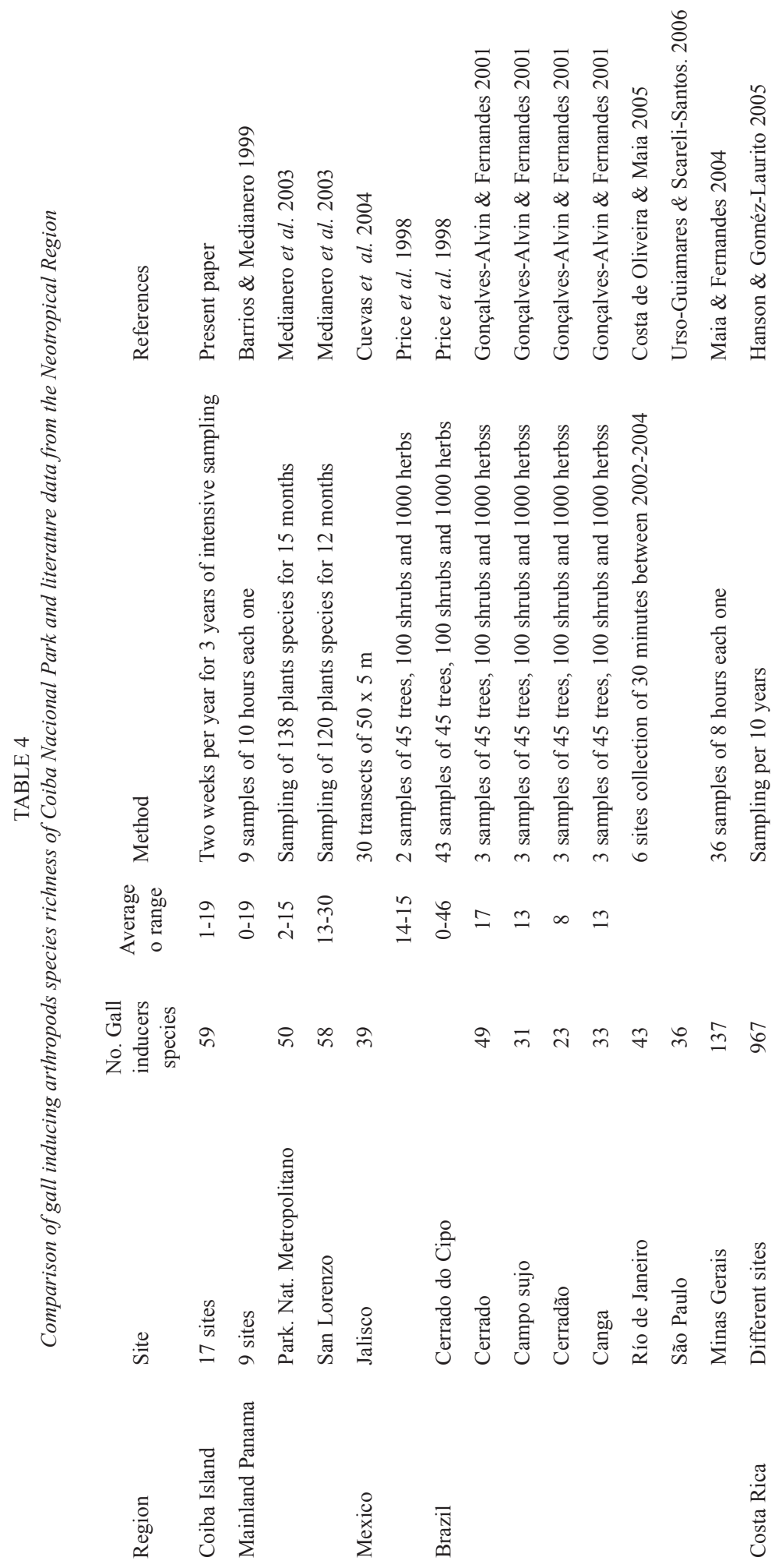


to that found at San Lorenzo $(\alpha=9.3)$, a six ha rain forest area on the Panamanian mainland (Medianero et al. 2003). Our results suggest that, according to insular biogeography theory (MacArthur and Wilson 1967), that Panama's continental territory, where close to 11000 species of plants have been identified (Correa 2001), as well as other sites in the Neotropical region, must be richer in arthropods than indicated by recorded data in literature. Certain climatic and edaphic factors can produce higher than expected gall-inducing species richness in certain warm temperature regions. Plant diversity is the predominant factor producing high gall-inducing species richness in wet tropical regions (Yukawa et al. 2001; Hanson \& Gómez-Laurito 2005). Our results support the idea that it may be premature to conclude that species richness of gall inducers declines near the equator (Hanson and Gómez-Laurito 2005). Furthermore there is an urgent need for more investigation of gall-inducing arthropods in the Neotropics, a region where as many as 20.000 gall-midge species (Cecidomyiidae), could exist (Espírito-Santo and Fernandes 2007).

\section{ACKNOWLEDGMENTS}

This work was supported by the Spanish Agency of International Cooperation (AECI) and also partially funded by the research project DGES PB97-1241, of the Spanish Ministry of Education and Culture to JLNA. Thanks to the Panamanian Authority of the Environment (ANAM) for collecting permits. We thank all the staff of AECI in Panama and ANAM in the Biological Research Station of Coiba, as well as many colleagues from The Natural Sciences Museum and the Royal Botanical Garden in Madrid, his friendly help and support in several ways who made possible this research. Special thanks to Dr. Santiago Castroviejo who was the direct responsible of conceiving and initiating the Coiba Inventory Research Project.

\section{RESUMEN}

El interés por el estudio de las agallas y los artrópodos que las inducen ha crecido en todo el mundo en los últimos veinte años. Sin embargo, los artrópodos que inducen agallas en la región Neotropical son probablemente los menos estudiados. Un estudio de la riqueza y composición de artrópodos que inducen agallas fue desarrollado en el Parque Nacional Coiba en la Republica de Panamá. Los datos provienen de muestreos intensivos de dos semanas, efectuados entre agosto de 1997 y septiembre de 1999, en 17 sitios del área insular del Parque Nacional Coiba. Un total de 4942 agallas, que corresponden a 50 especies de insectos y nueve de ácaros, fueron colectadas en 50 especies de plantas vasculares de 30 familias. El $62.7 \%$ de las agallas correspondieron a especies de la familia Cecidomyiidae (Diptera), el 15.3\% a ácaros de la familia Eriophyidae, el $8.5 \%$ a Psyllidae (Homoptera), un 6.8\% a Coccidae y el $5.1 \%$ a Phlaeothripidae (Tysanoptera). Las familias de plantas con más especies de inductores de agallas fueron Myrtaceae con siete, Bignoniaceae con cinco y Euphorbiaceae, Fabaceae y Melastomataceae con cuatro. Las agallas formadas en hojas representaron el 93\% del total. El número de inductores de agallas por sitio osciló entre uno y diecinueve. La riqueza de artrópodos inductores de agallas del Parque Nacional Coiba se discute con datos disponibles de la literatura para el área continental de Panamá y el Neotrópico. Nuestros resultados apoyan la idea de que es prematuro concluir que la riqueza de artrópodos que inducen agallas disminuye hacia el Ecuador.

Palabras clave: Coiba, insectos gallícolas, riqueza de agallas, plantas hospedadoras, interacción planta/insecto, Panamá.

\section{REFERENCES}

Ananthakrishnan, T.N. 1984. Adaptive strategies in cecidogenous insect, p. 1-9. In T. N. Ananthakrishnan (ed.). The Biology of gall insect. Oxford \& IBH, New Delhi, India. 362 p.

Barrios, H. \& E. Medianero. 1999. Riqueza de insectos formadores de agallas en nueve zonas de vida de la Republica de Panamá. Scientia 14:39-58.

Castroviejo, S. 1997. Introducción y consideraciones biogeográficas, p 1-8 In S. Castroviejo (ed.). Flora y fauna del Parque Nacional Coiba (Panamá). Spanish Agency for International Cooperation, Madrid, Spain.

Colwell, R. K. 2006. EstimateS: statistical estimation of species richness and shared species from samples (Software and User's Guide). Version 8. http://viceroy.eeb.uconn.edu/estimates.

Correa, M. 2001. Diversidad en la Flora de Panamá. p. 70-75. In S. Heckadon-Moreno (ed.). Panamá: Puente biologico. Smithsonian Tropical Research Institute, Balboa, Panama. 
Costa De Oliveira, J. \& V. C. Maia. 2005. Presencia y caracterizaçao de galhas de insetos na restinga de Grumari (Rio de Janeiro RJ, Brazil). Arquitos do Museo Nacional, Rio de Janeiro 63: 669-675.

Cuevas-Reyes, P., M. Quesada, P. Hanson, R. Dirzo \& K. Oyama. 2004a. Diversity of gall-inducing insects in a Mexican tropical dry forest: the importance of plant species richness, life-forms, host plant age and plant density. J. Ecol. 92:707-716.

Cuevas-Reyes, P., M. Quesada, C. Siebe, \& K. Oyama. 2004b. Spatial patterns of herbivory by gall-forming insects: a test of the soil fertility in a Mexican tropical dry forest. Oikos 107: 181-189.

Espírito-Santo, M.M. \& G.W. Fernandes. 2007. How many species of gall-inducing insects are there on Earth, and Where are they. Ann Entomol Soc Am. 100: 95-99.

Felt, E.P. 1940. Plant galls and gall inducers. Comstock, New York, New York, USA. 364 p.

Fontal, F. \& J.L. Nieves-Aldrey. 2004. Estudio comparativo de diversidad de eucoilidos paleárticos (El Ventorrillo, España) y neotropicales (P.N. Coiba, Panamá) (Hymenoptera, Cynipoidea, Figitidae, Eucoilinae). Boletín de la Sociedad Entomológica Aragonesa 35:51-101.

Gagné, R.J. 1984. The geography of gall insects, p 305322. In T.N. Ananthakrishnan (ed.) The Biology of gall insect. Oxford and IBH, New Delhi, India.

Gagné, R.J. 1994. The Gall Midges of the Neotropical. Cornell University, Ithaca, New. York, USA. 352 p.

Gonçalves-Alvim, S.J. \& G.W. Fernandes. 2001. Biodiversity of galling insect: historical, community and habitat effects in four neotropical savannas. Biodivers Conserv. 10: 79-98.

Gotelli, N.J. \& R.K. Colwell. 2001. Quantifying biodiversity: procedures and pitfalls in the measurement and comparison of species richness. Ecol Lett. 4: 379-391.

Hanson, P.E. \& J. Gomez-Laurito. 2005. Diversity of gall-inducing arthropods of Costa Rica, p 673692 In A. Raman, C. Schaefer \& T. Withers (eds.) Biology, ecology, and evolution of gall-inducing arthropods. Science, Enfield, New Hampshire, USA. 817 p.

Houard, C. 1908-1913. Les Zoocécidies des Plantes d'Europe el du Bassin de la Mediterranée. 3 Tome. Librairie Scientifique A. Hermann, Paris, France.
Houard, C. 1922. Les Zoocécidies des Plantes d'Afrique, d'Asie et d'Océanie. 2 Tome. Librairie Scientifique Jules Hermann, Paris, France.

Houard, C. 1933. Les Zoocécidies des Plantes de l'Amerique du Sud et de l'Amerique Centrale. Librairie Scientifique A. Hermann, Paris, France.

Houard, C. 1940. Les Zoocécidies des Plantes de l'Amerique du Nord. Librairie Scientifique A. Hermann, Paris, France.

Macarthur, R.H. \& E.O. Wilson. 1967. The Theory of Island Biogeography. Princeton University, New Jersey, USA.

Maia, V.C. 2001. The gall midges (Diptera, Cecidomyiidae) fron three restingas of Rio de Janeiro State, Brazil. Rev. Bras. Zool. 18: 583-629.

Maia, V.C. 2005. Catálogo dos Cecidomyiidae (Diptera) do estado do Rio de Janeiro. Biota Neotropical 5: 1-15.

Maia, V.C. \& G.W. Fernandes. 2004. Insect gall from Serra de São José (Tiradentes, MG, Brazil). Braz J Biol. 64: 423-445.

Mani, M.S. 1964. The ecology of plant galls. Dr. W. Junk, Publishers, The Hague, Netherlands.

Mani, M.S. 1992. Introduction to Cecidology. p 3-7. In J. D. Shorthouse \& O. Rohfritsh, (eds.) Biology of Insect-Induced Galls. Oxford Univ, New York, USA.

Medianero, E., A. Valderrama \& H. Barrios 2003. Diversidad de insectos minadores de hojas y formadores de agallas en el dosel y sotobosque del bosque tropical. Acta Zool Mex. 89:153:168.

Meyer, J. 1987. Plant galls and gall inducers. Gebrüider Borntraeger Verlagsbuchhandlung Science, Berlin, Germany. 291 p.

Nieves-Aldrey, J.L. 1998. Insectos que inducen la formación de agallas en las plantas; una fascinante interacción ecológica y evolutiva. Bol Soc Entomol Aragonesa 23:3-12.

Price, P., G.W. Fernandes, A.C. F. Lara, J. Brawn, H. Barrios, M.G. Wright, S. Ribeiro, \& N. Rothcliff. 1998. Global patterns in local number of insects galling species. J Biogeogr. 25:581-591.

Raman, A., C.W. Schaefer \& T.M. Withers. 2005. Galls and gall-inducing arthropods: an overview of their Biology, Ecology and Evolution. p 1-33 In A. Raman, C. Schaefer \& T. Withers (eds.) Biology, ecology, and evolution of gall-inducing Arthropods. Science, Enfield, New Hampshire, USA. 817 p. 
Schlinger, E.I. 1974. Continental drift, Nothofagus, and some ecologically associated insects. Ann Rev Entomol.19:323-343.

Stone, G.N. \& N. Schönrogge. 2003. The adaptive significance of insect gall morphology. Trends Ecol Evol. 18:512-522.

Urso-Guimares, M.V. \& C. Scareli-Santos. 2006. Gall and gall inducers in plants from the Pé-de-Gigate Cerrado
Reserve, Santa Rita do Passa Quatro SP, Brazil. Braz J Biol. 66:357-369.

Wolda, H. 1983. Diversidad de la entomofauna y cómo medirla. Informe Final IX Claz Perú: 181-186

Yukawa, J., M. Tokuda, N. Uechi \& S. Sato. 2001a. Species richness of galling arthropods in Manaus Amazon and the Surroundings of the Iguassus Falls. Esakia 41:11-15. 